\title{
Generalized System of Variational Inequalities in Banach Spaces
}

\author{
Abdellah Bnouhachem ${ }^{1,2, *}$, Muhammad Aslam Noor ${ }^{3}$, Mohamed Khalfaoui $^{4}$ and Hafida Benazza \\ ${ }^{1}$ School of Management Science and Engineering, Nanjing University, Nanjing, 210093, P.R. China \\ 2 Ibn Zohr University, ENSA, BP 1136, Agadir, Morocco \\ ${ }^{3}$ Mathematics Department, COMSATS Institute of Information Technology, Islamabad, Pakistan \\ ${ }^{4}$ Ecole Supérieure de Technologie de Salé, Mohamed 5 University, Agdal-Rabat, Morocco
}

Received: 9 May. 2013, Revised: 13 Sep. 2013, Accepted: 14 Sep. 2013

Published online: 1 May. 2014

\begin{abstract}
In this paper, we introduce and consider a new system of extended general variational inequalities in Banach spaces. We establish the equivalence between the extended general variational inequalities and the fixed point problems. We use this equivalent formulation to suggest some iterative methods for solving this new system. We prove the convergence analysis of the proposed iterative methods under some suitable conditions. Several special cases, which can be obtained from main results are discussed. The idea and technique of this paper may stimulate further research activities in these fields.
\end{abstract}

Keywords: Sunny nonexpansive retraction, generalized variational inequalities system, $q$ - uniformly smooth Banach spaces, iterative algorithm, relaxed cocoercive mappings.

2010 AMS Subject Classification: 49J40, 65N30

\section{Introduction}

Variational inequality and complementarity problems are of fundamental importance in a wide range of mathematical and applied sciences problems, such as mathematical programming, traffic engineering, economics and equilibrium problems, see [1-18]. The ideas and techniques of the variational inequalities are being applied in a variety of diverse areas of sciences and proved to be productive and innovative. It has been shown that this theory provides a simple, natural and unified framework for a general treatment of unrelated problems. The fixed-point theory has played an important role in the development of various algorithms for solving variational inequalities. Using the projection operator technique, one usually establishes an equivalence between the variational inequalities and the fixed-point problem. This alternative equivalent formulation was used by Lions and Stampacchia [6] to study the existence of a solution of the variational inequalities. Projection methods and its variant forms represent important tools for finding the approximate solution of variational inequalities. We now have a variety of techniques to suggest and analyze various iterative algorithms for solving variational inequalities and the related optimization problems. In recent years, some new and interesting problems, which are called the system of variational inclusions were introduced and studied. Chang et al. [2], Huang and Noor [5], Noor and Noor [10], Noor [9], Verma [18, 19,20] and Yang et al. [22] introduced and studied a system of variational inclusions involving four, three, two different nonlinear operators.

Inspired and motivated by research going on in this area, we introduce and consider a new system of extended general variational inequalities involving six different nonlinear operators in Banach spaces. We establish the equivalence between this system of extended general variational inequalities and the fixed point problems using the projection operator technique. This equivalent formulation is used to suggest and analyze some new iterative methods for solving the extended general variational inequalities. We also prove the convergence analysis of the proposed algorithm under some suitable mild conditions. Since this class of systems includes the system of variational inequalities involving four, three,

\footnotetext{
*Corresponding author e-mail: babedallah@yahoo.com
} 
two operators and the classical variational inequalities as special cases, results obtained in this paper continue to hold for these problems. It is expected that these results may inspire and motivate others to find novel and innovative applications in various branches of pure and applied sciences.

The readers are encouraged to explore the novel and innovative applications of the system of extended general variational inequalities and its variant forms in different areas of pure and applied sciences.

\section{Preliminaries}

Throughout this article, let $X$ be a real Banach space with its dual space $X^{*}$. We usually use $\langle\cdot, \cdot\rangle$ to denote the pairing between $X$ and $X^{*}$, and $2^{X}$ denote the family of all the nonempty subsets of $X$. The generalized duality mapping $J_{q}(x): X \longrightarrow 2^{X^{*}}$ is defined by

$$
J_{q}(x)=\left\{f^{*} \in X^{*}:\left\langle x, f^{*}\right\rangle=\|x\|^{q},\left\|f^{*}\right\|=\|x\|^{q-1}\right\},
$$

where $q>1$ is a constant. In particular, $J_{2}=J$ is the usual normalized duality mapping. It is known that, in general, $J_{q}=\|x\|^{q-2} J_{2}$, for all $x \neq 0$, and $J_{q}(x)$ is single-valued if $X^{*}$ is strictly convex. If $X=H$ is a Hilbert space then $J_{2}$ becomes the identity mapping of $H$. Note that $X$ is a uniformly smooth Banach space if and only if $J_{q}$ is singlevalued and uniformly continuous on any bounded subset of $X$.

Let $K$ be a nonempty, closed and convex subset of $X$. A mapping $Q: X \longrightarrow K$ is said to be sunny if

$$
Q(Q(x)+t(x-Q(x)))=Q(x), \forall x \in X, \forall t \geq 0 .
$$

A mapping $Q: X \longrightarrow K$ is said to be a retraction or a projection if $Q(x)=x ; \forall x \in K$. If $X$ is smooth then the sunny nonexpansive retraction of $X$ onto $K$ is uniquely decided (see [4]). Using $J_{q}=\|x\|^{q-2} J_{2}$, replace $J$ by $J_{q}$, it is easy to obtain the following results by Bruck [1], Goebel and Reich [3].

Proposition 2.1 Let $X$ be a $q$-uniformly smooth Banach spaces and let $K$ be a nonempty subset of $X$. Let $Q_{K}: X \longrightarrow K$ be a retraction and let $J_{q}$ be the generalized duality mapping on $X$. Then the following are equivalent:

(a) $Q_{K}$ is sunny and nonexpansive.

(b) $\left\|Q_{K}(x)-Q_{K}(y)\right\|^{2}$

$\leq\left\langle x-y, J_{q}\left(Q_{K}(x)-Q_{K}(y)\right)\right\rangle, \forall x, y \in X$.

(c) $\left\langle x-Q_{K}(x), J_{q}\left(Q_{K}(x)-y\right)\right\rangle \geq 0, \forall y \in K$.

Lemma 2.1 ( See [21]) Let $X$ be a real $q$-uniformly smooth Banach space $(q>1)$, then there exists a constant $c_{q}>0$ such that

$$
\|x+y\|^{q} \leq\|x\|^{q}+q\left\langle y, J_{q} x\right\rangle+c_{q}\|y\|^{q}, \forall x, y \in X .
$$

In particular, if $X$ be a real 2-uniformly smooth Banach space, then there exists a constant $c_{2}>0$ such that

$$
\|x+y\|^{2} \leq\|x\|^{2}+2\left\langle y, J_{2} x\right\rangle+c_{2}\|y\|^{2}, \forall x, y \in X .
$$

Let $K$ be a nonempty, closed and convex subset of $X$. For given nonlinear operators $T_{i}(.,):. X \times X \longrightarrow X$ and $g_{i}, h_{i}: X \longrightarrow X(i=1,2)$, we consider problem of finding $\left(x^{*}, y^{*}\right) \in X \times X:\left(h_{1}\left(x^{*}\right), h_{2}\left(y^{*}\right)\right) \in K \times K$ such that

$$
\left.\begin{array}{l}
\left\langle\rho T_{1}\left(y^{*}, x^{*}\right)+h_{1}\left(x^{*}\right)-g_{1}\left(y^{*}\right),\right. \\
\left.J_{q}\left(g_{1}(x)-h_{1}\left(x^{*}\right)\right)\right\rangle \geq 0 ; \forall x \in X, g_{1}(x) \in K \text { and } \rho>0, \\
\left\langle\eta T_{2}\left(x^{*}, y^{*}\right)+h_{2}\left(y^{*}\right)-g_{2}\left(x^{*}\right),\right. \\
\left.J_{q}\left(g_{2}(x)-h_{2}\left(y^{*}\right)\right)\right\rangle \geq 0 ; \forall x \in X, g_{2}(x) \in K \text { and } \eta>0 .
\end{array}\right\}
$$

which is called the system of extended general variational inequalities.

We now discuss some applications of the system of extended general variational inequalities (1).

If $X=H$ is a Hilbert space, $J_{q}=I, h_{i}=I$, the identity operator, then problem (1) reduces to finding $\left(x^{*}, y^{*}\right) \in K \times K$ such that

$$
\left.\begin{array}{l}
\left\langle\rho T_{1}\left(y^{*}, x^{*}\right)+x^{*}-g_{1}\left(y^{*}\right),\right. \\
\left.g_{1}(x)-x^{*}\right\rangle \geq 0 ; \\
\forall x \in H, g_{1}(x) \in K \text { and } \rho>0, \\
\left\langle\eta T_{2}\left(x^{*}, y^{*}\right)+y^{*}-g_{2}\left(x^{*}\right), g_{2}(x)-y^{*}\right\rangle \geq 0 ; \\
\forall x \in H, g_{2}(x) \in K \text { and } \eta>0,
\end{array}\right\}
$$

which were introduced and studied by Noor and Noor [10].

If $g_{i}=g(i=1,2)$, then the system (2) is equivalent to finding $\left(x^{*}, y^{*}\right) \in K \times K$ such that

$$
\left.\begin{array}{l}
\left\langle\rho T_{1}\left(y^{*}, x^{*}\right)+x^{*}-g\left(y^{*}\right), g(x)-x^{*}\right\rangle \geq 0 ; \\
\forall x \in H, g(x) \in K \quad \text { and } \quad \rho>0, \\
\left\langle\eta T_{2}\left(x^{*}, y^{*}\right)+y^{*}-g\left(x^{*}\right), g(x)-y^{*}\right\rangle \geq 0 ; \\
\forall x \in H, g(x) \in K \quad \text { and } \quad \eta>0,
\end{array}\right\}
$$

which was considered and investigated by Noor [9].

If $X=H$ is a Hilbert space, $J_{q}=I, T_{i}(x, y)=T_{i}(x)$, $h_{i}=g_{i}=g(i=1,2)$, then problem (1) reduces to finding $\left(x^{*}, y^{*}\right) \in H \times H:\left(g\left(x^{*}\right), g\left(y^{*}\right)\right) \in K \times K$ such that

$$
\left.\begin{array}{l}
\left\langle\rho T_{1}\left(y^{*}\right)+g\left(x^{*}\right)-g\left(y^{*}\right), g(x)-g\left(x^{*}\right)\right\rangle \geq 0 ; \\
\forall x \in H, g(x) \in K \quad \text { and } \rho>0, \\
\left\langle\eta T_{2}\left(x^{*}\right)+g\left(y^{*}\right)-g\left(x^{*}\right), g(x)-g\left(y^{*}\right)\right\rangle \geq 0 ; \\
\forall x \in H, g_{2}(x) \in K \quad \text { and } \quad \eta>0,
\end{array}\right\}
$$

which has been introduced and studied by Yang et al. [22].

If $X=H$ is a Hilbert space, $x^{*}=y^{*}, J_{q}=I, T_{i}(x, y)=$ $T_{1}(x)=T_{2}(x)(i=1,2), h_{1}=h_{2}, g_{1}=g_{2}$, then problem (1) reduces to finding $x^{*} \in H: h_{1}\left(x^{*}\right) \in K \times K$ such that

$\left\langle\rho T_{1}\left(x^{*}\right)+h_{1}\left(x^{*}\right)-g_{1}\left(x^{*}\right), g_{1}(x)-h_{1}\left(x^{*}\right)\right\rangle \geq 0 ;$

$\forall x \in H: g_{1}(x) \in K \quad$ and $\rho>0$,

which is called the extended general variational inequality, introduced and studied by Noor [11] in 2009. For the applications, numerical results, generalizations and other aspects of extended general variational inequalities, see $[11,12,13,14]$ and the references therein. 
If $g=I$, then problem (3) reduces of finding $\left(x^{*}, y^{*}\right) \in$ $K \times K$ such that

$$
\left.\begin{array}{l}
\left\langle\rho T_{1}\left(y^{*}, x^{*}\right)+x^{*}-y^{*}, x-x^{*}\right\rangle \geq 0 ; \\
\forall x \in K \text { and } \rho>0, \\
\left\langle\eta T_{2}\left(x^{*}, y^{*}\right)+y^{*}-x^{*}, x-y^{*}\right\rangle \geq 0 ; \\
\forall x \in K \text { and } \eta>0,
\end{array}\right\}
$$

which has been considered and studied by Huang and Noor [5].

If $T_{1}=T_{2}=T$, then problem (6) reduces of finding $\left(x^{*}, y^{*}\right) \in K \times K$ such that

$$
\left.\begin{array}{l}
\left\langle\rho T\left(y^{*}, x^{*}\right)+x^{*}-y^{*}, x-x^{*}\right\rangle \geq 0 ; \\
\forall x \in K \text { and } \rho>0, \\
\left\langle\eta T\left(x^{*}, y^{*}\right)+y^{*}-x^{*}, x-y^{*}\right\rangle \geq 0 ; \\
\forall x \in K \text { and } \eta>0,
\end{array}\right\}
$$

The system (7) has been studied and investigated by Chang et al. [2] and Verma [19].

If $T_{1}=T_{2}=T$, and $g=I$, then problem (4) reduces of finding $\left(x^{*}, y^{*}\right) \in K \times K$ such that

$$
\left.\begin{array}{l}
\left\langle\rho T\left(y^{*}\right)+x^{*}-y^{*}, x-x^{*}\right\rangle \geq 0 ; \\
\forall x \in K \text { and } \rho>0, \\
\left\langle\eta T\left(x^{*}\right)+y^{*}-x^{*}, x-y^{*}\right\rangle \geq 0 ; \\
\forall x \in K \text { and } \eta>0,
\end{array}\right\}
$$

which has been introduced and studied by Verma [18,20].

If $x^{*}=y^{*}$, then problem (8) collapses to finding $x^{*} \in K$. such that

$$
\left\langle T\left(x^{*}\right), x-x^{*}\right\rangle \geq 0, \quad \forall x \in K .
$$

Inequality of type (9) is called variational inequality, which was introduced and studied by Stampacchia [17] in 1964. The system of extended general variational inequalities (1) includes several classes of variational inequalities and related optimization problems as special cases. For the recent research, see [1-18] and the references therein.

We now recall some well-known results and concepts, which are needed.

Definition 2.1 A mapping $h: X \longrightarrow X$ is called

(a) $r$-strongly monotone if, there exists a constant $r>0$ such that

$$
\left\langle h(x)-h(y), J_{q}(x-y)\right\rangle \geq r\|x-y\|^{2}, \forall x, y \in X ;
$$

(b) $(\xi, \varsigma)$-relaxed cocoercive if, there exist constants $\xi, \varsigma>0$ such that

$$
\begin{aligned}
\left\langle h(x)-h(y), J_{q}(x-y)\right\rangle & \geq-\xi\|h(x)-h(y)\|^{2} \\
& +\varsigma\|x-y\|^{2}, \forall x, y \in X ;
\end{aligned}
$$

(c) $\gamma$-Lipschitz continuous if, there exists a constant $\gamma>0$ such that

$$
\|h(x)-h(y)\| \leq \gamma\|x-y\|, \forall x, y \in X .
$$

Definition 2.2 Let $T: X \times X \longrightarrow X$ and $g: X \longrightarrow X$. Then $T$ is called

(a) $r$-strongly monotone with respect to $g$ if, there exists a constant $r>0$ such that

$$
\begin{aligned}
& \left\langle T\left(x_{1}, y\right)-T\left(x_{2}, y\right), J_{q}\left(g\left(x_{1}\right)-g\left(x_{2}\right)\right)\right\rangle \\
& \geq r\left\|x_{1}-x_{2}\right\|^{2}, \forall x_{1}, x_{2}, y \in X
\end{aligned}
$$

(b) $(\xi, \varsigma)$-relaxed cocoercive with respect to $g$ if, there exist constants $\xi, \varsigma>0$ such that

$$
\begin{aligned}
& \left\langle T\left(x_{1}, y\right)-T\left(x_{2}, y\right), J_{q}\left(g\left(x_{1}\right)-g\left(x_{2}\right)\right)\right\rangle \\
& \geq-\xi\left\|T\left(x_{1}, y\right)-T\left(x_{2}, y\right)\right\|^{2}+\varsigma\left\|x_{1}-x_{2}\right\|^{2}, \\
& \forall x_{1}, x_{2}, y \in X ;
\end{aligned}
$$

(c) $\gamma$-Lipschitz continuous in the first variable if, there exists a constant $\gamma>0$ such that

$$
\left\|T\left(x_{1}, y\right)-T\left(x_{2}, y\right)\right\| \leq \gamma\left\|x_{1}-x_{2}\right\|, \forall x_{1}, x_{2}, y \in X .
$$

(d) $\gamma$-Lipschitz continuous in the second variable if, there exists a constant $\gamma>0$ such that

$$
\left\|T\left(x, y_{1}\right)-T\left(x, y_{2}\right)\right\| \leq \gamma\left\|y_{1}-y_{2}\right\|, \forall x, y_{1}, y_{2} \in X .
$$

\section{Main Results}

In this section, we first establish the equivalence between the system of the extended general variational inequality (1) and the fixed point problems. Then by using the obtained fixed point formulation, we construct a new iterative algorithm for solving the systems (1).

Lemma 3.1 Let $X$ be an smooth Banach space, for given nonlinear operators $T_{i}: X \times X \longrightarrow X, g_{i}, h_{i}: X \longrightarrow X(i=$ $1,2)$, and $\rho, \eta>0$. Then the point $\left(x^{*}, y^{*}\right) \in K \times K$ is a solution of (1) if and only if

$$
\left.\begin{array}{l}
h_{1}\left(x^{*}\right)=Q_{K}\left[g_{1}\left(y^{*}\right)-\rho T_{1}\left(y^{*}, x^{*}\right)\right] \\
h_{2}\left(x^{*}\right)=Q_{K}\left[g_{2}\left(x^{*}\right)-\eta T_{2}\left(x^{*}, y^{*}\right)\right] .
\end{array}\right\}
$$

Proof. The first variational inequality of (1) can be written as follows:

$$
\begin{aligned}
& \left\langle\left[g_{1}\left(y^{*}\right)-\rho T_{1}\left(y^{*}, x^{*}\right)\right]-h_{1}\left(x^{*}\right), J_{q}\left(h_{1}\left(x^{*}\right)-g_{1}(x)\right)\right\rangle \geq 0 ; \\
& \forall x \in X, g_{1}(x) \in K \quad \text { and } \quad \rho>0 .
\end{aligned}
$$

We can deduce from Proposition 2.1 (c) that the above inequality is equivalent to

$$
h_{1}\left(x^{*}\right)=Q_{K}\left[g_{1}\left(y^{*}\right)-\rho T_{1}\left(y^{*}, x^{*}\right)\right] .
$$

Similar, the second variational inequality of (1) is equivalent to

$$
h_{2}\left(x^{*}\right)=Q_{K}\left[g_{2}\left(x^{*}\right)-\eta T_{2}\left(x^{*}, y^{*}\right)\right] .
$$

Lemma 3.1 implies that the system of extended general variational inequalities (1) and the fixed point problems 
(10) are equivalent. This equivalent formulation plays a crucial role in developing the iterative methods for solving (1). We rewrite (10) in the following form:

$$
\left\{\begin{array}{l}
h_{1}(x)=Q_{K}[z] \\
h_{2}(y)=Q_{K}[t] \\
z=(1-\alpha) z+\alpha\left[g_{1}(y)-\rho T_{1}(y, x)\right] \\
t=(1-\alpha) t+\alpha\left[g_{2}(x)-\eta T_{2}(x, y)\right],
\end{array}\right.
$$

which enables to suggest the following iterative method,

Algorithm 3.1 Let $T_{i}, g_{i}, h_{i}(i=1,2)$, be nonlinear operators, $\rho, \eta>0$. For arbitrary chosen initial points $z^{0}, t^{0} \in K$, compute the iterative sequence $\left\{\left(x^{k}, y^{k}\right\}\right.$ by using

$$
\left.\begin{array}{l}
h_{1}\left(x^{k}\right)=Q_{K}\left[z^{k}\right] \\
h_{2}\left(y^{k}\right)=Q_{K}\left[t^{k}\right] \\
z^{k+1}=(1-\alpha) z^{k}+\alpha\left[g_{1}\left(y^{k}\right)-\rho T_{1}\left(y^{k}, x^{k}\right)\right] \\
t^{k+1}=(1-\alpha) t^{k}+\alpha\left[g_{2}\left(x^{k}\right)-\eta T_{2}\left(x^{k}, y^{k}\right)\right],
\end{array}\right\}
$$

where $Q_{K}$ is the sunny nonexpansive retraction of $X$ onto $K$ and $0<\alpha \leq 1$.

We now establish the strongly convergence of the sequences generated by Algorithm 3.1.

Theorem 3.1 Let $T_{i}, g_{i}, h_{i}(i=1,2)$, be nonlinear operators, such that for each $\mathrm{i}=1,2$,

(a) $T_{i}$ is $\left(\kappa_{i}, \theta_{i}\right)$-relaxed cocoercive with respect to $g_{i}$ and $\gamma_{i}$-Lipschitz continuous in the first variable, and $\mu_{i}$-Lipschitz continuous in the second variable;

(b) $h_{i}$ is $\left(\xi_{i}, v_{i}\right)$-relaxed cocoercive and $\delta_{i}$-Lipschitz continuous;

(c) $g_{i}$ is $\sigma_{i}$-Lipschitz continuous.

If there exist constants $\rho, \eta>0$ such that

$$
\left.\begin{array}{l}
\sqrt[q]{\left(\sigma_{2}^{q}-\eta q \theta_{2}\right)+\left(\eta q \kappa_{2}+C_{q} \eta^{q}\right) \gamma_{2}^{q}} \\
<1-k_{1}-\rho \mu_{1}, k_{1}+\rho \mu_{1}<1 \\
\sqrt[q]{\left(\left(\sigma_{1}^{q}-\rho q \theta_{1}\right)+\left(\rho q \kappa_{1}+C_{q} \rho^{q}\right) \gamma_{1}^{q}\right)} \\
<1-k_{2}-\eta \mu_{2}, k_{2}+\eta \mu_{2}<1, \\
\text { and } \\
q v_{i}<1+\left(C_{q}+q \xi_{i}\right) \delta_{i}^{q},(i=1,2), \\
\text { where } \\
k_{i}=\sqrt[q]{\left(1-q v_{i}\right)+\left(C_{q}+q \xi_{i}\right) \delta_{i}^{q}},(i=1,2),
\end{array}\right\}
$$

then for arbitrarily chosen initial points $x_{0}, y_{0} \in K, x^{k}$ and $y^{k}$ obtained from Algorithm 3.1 converge strongly to $x^{*}$ and $y^{*}$ respectively.

Proof. It follows from (11) that

$$
\begin{aligned}
& \left\|z^{k+1}-z^{k}\right\| \\
= & \|(1-\alpha) z^{k}+\alpha\left[g_{1}\left(y^{k}\right)-\rho T_{1}\left(y^{k}, x^{k}\right)\right]-(1-\alpha) z^{k-1} \\
- & \alpha\left[g_{1}\left(y^{k-1}\right)-\rho T_{1}\left(y^{k-1}, x^{k-1}\right)\right] \\
\leq & (1-\alpha)\left\|z^{k}-z^{k-1}\right\|+\alpha \| g_{1}\left(y^{k}\right)-g_{1}\left(y^{k-1}\right) \\
- & \rho\left(T_{1}\left(y^{k}, x^{k}\right)-T_{1}\left(y^{k-1}, x^{k-1}\right)\right) \|
\end{aligned}
$$

From $T_{1} \mu_{1}$-Lipschitz continuous in the second variable, we have

$$
\left\|g_{1}\left(y^{k}\right)-g_{1}\left(y^{k-1}\right)-\rho\left(T_{1}\left(y^{k}, x^{k}\right)-T_{1}\left(y^{k-1}, x^{k-1}\right)\right)\right\|
$$

$$
\begin{aligned}
& \leq\left\|g_{1}\left(y^{k}\right)-g_{1}\left(y^{k-1}\right)-\rho\left(T_{1}\left(y^{k}, x^{k}\right)-T_{1}\left(y^{k-1}, x^{k}\right)\right)\right\| \\
& \left.+\rho \| T_{1}\left(y^{k-1}, x^{k}\right)-T_{1}\left(y^{k-1}, x^{k-1}\right)\right) \| \\
& \leq\left\|g_{1}\left(y^{k}\right)-g_{1}\left(y^{k-1}\right)-\rho\left(T_{1}\left(y^{k}, x^{k}\right)-T_{1}\left(y^{k-1}, x^{k}\right)\right)\right\| \\
& +\rho \mu_{1}\left\|x^{k}-x^{k-1}\right\|
\end{aligned}
$$

Since $T_{1}$ is $\left(\kappa_{1}, \theta_{1}\right)$-relaxed cocoercive with respect to $g_{1}$ and $\gamma_{1}$-Lipschitz continuous in the first variable, and $g_{1}$ is $\sigma_{1}$-Lipschitz continuous, we can conclude that

$$
\begin{aligned}
& \left\|g_{1}\left(y^{k}\right)-g_{1}\left(y^{k-1}\right)-\rho\left(T_{1}\left(y^{k}, x^{k}\right)-T_{1}\left(y^{k-1}, x^{k}\right)\right)\right\|^{q} \\
= & \left\|g_{1}\left(y^{k}\right)-g_{1}\left(y^{k-1}\right)\right\|^{q}-\rho q\left\langle T_{1}\left(y^{k}, x^{k}\right)-T_{1}\left(y^{k-1}, x^{k}\right),\right. \\
& \left.J_{q}\left(g_{1}\left(y^{k}\right)-g_{1}\left(y^{k-1}\right)\right)\right\rangle \\
+ & C_{q} \rho^{q}\left\|T_{1}\left(y^{k}, x^{k}\right)-T_{1}\left(y^{k-1}, x^{k}\right)\right\|^{q} \\
\leq & \left\|g_{1}\left(y^{k}\right)-g_{1}\left(y^{k-1}\right)\right\|^{q} \\
+ & \rho q \kappa_{1}\left\|T_{1}\left(y^{k}, x^{k}\right)-T_{1}\left(y^{k-1}, x^{k}\right)\right\|^{q}-\rho q \theta_{1}\left\|y^{k}-y^{k-1}\right\|^{q} \\
+ & C_{q} \rho^{q}\left\|T_{1}\left(y^{k}, x^{k}\right)-T_{1}\left(y^{k-1}, x^{k}\right)\right\|^{q} \\
\leq & \left(\left(\sigma_{1}^{q}-\rho q \theta_{1}\right)+\left(\rho q \kappa_{1}+C_{q} \rho^{q}\right) \gamma_{1}^{q}\right)\left\|y^{k}-y^{k-1}\right\|^{q}
\end{aligned}
$$

Substituting (14) and (15) in (13), we get

$$
\begin{aligned}
& \left\|z^{k+1}-z^{k}\right\| \\
\leq & (1-\alpha)\left\|z^{k}-z^{k-1}\right\|+\alpha \sqrt[q]{(}\left(\sigma_{1}^{q}-\rho q \theta_{1}\right) \\
+ & \left.\left(\rho q \kappa_{1}+C_{q} \rho^{q}\right) \gamma_{1}^{q}\right)\left\|y^{k}-y^{k-1}\right\| \\
+ & \alpha \rho \mu_{1}\left\|x^{k}-x^{k-1}\right\| .
\end{aligned}
$$

In a similar way, we can prove that

$$
\begin{aligned}
& \left\|t^{k+1}-t^{k}\right\| \\
\leq & (1-\alpha)\left\|t^{k}-t^{k-1}\right\| \\
+ & \alpha \sqrt[q]{\left(\left(\sigma_{2}^{q}-\eta q \theta_{2}\right)+\left(\eta q \kappa_{2}+C_{q} \eta^{q}\right) \gamma_{2}^{q}\right)}\left\|x^{k}-x^{k-1}\right\| \\
+ & \alpha \eta \mu_{2}\left\|y^{k}-y^{k-1}\right\| .
\end{aligned}
$$

On the other hand, by using (11), we find that

$$
\begin{aligned}
& \left\|x^{k}-x^{k-1}\right\| \\
\leq & \left\|x^{k}-x^{k-1}-\left(h_{1}\left(x^{k}\right)-h_{1}\left(x^{k-1}\right)\right)\right\| \\
+ & \left\|a_{k}\left(z^{k}\right)-Q_{k}\left(x^{k-1}\right)\right\| \\
\leq & \left\|x^{k}-x^{k-1}-\left(h_{1}\left(x^{k}\right)-h_{1}\left(x^{k-1}\right)\right)\right\|+\left\|z^{k}-z^{k-1}\right\| .
\end{aligned}
$$

From $\left(\xi_{1}, v_{1}\right)$-relaxed cocoercive and $\delta_{1}$-Lipschitz continuous of $h_{1}$, we have

$$
\begin{aligned}
& \left\|x^{k}-x^{k-1}-\left(h_{1}\left(x^{k}\right)-h_{1}\left(x^{k-1}\right)\right)\right\|^{q} \\
\leq & \left\|x^{k}-x^{k-1}\right\|^{q}-q\left\langle h_{1}\left(x^{k}\right)-h_{1}\left(x^{k-1}\right), J_{q}\left(x^{k}-x^{k-1}\right)\right\rangle \\
& +C_{q}\left\|h_{1}\left(x^{k}\right)-h_{1}\left(x^{k-1}\right)\right\|^{q} \\
\leq & \left\|x^{k}-x^{k-1}\right\|^{q}+q \xi_{1}\left\|h_{1}\left(x^{k}\right)-h_{1}\left(x^{k-1}\right)\right\|^{q} \\
- & q v_{1}\left\|x^{k}-x^{k-1}\right\|^{q} \\
& +C_{q}\left\|h_{1}\left(x^{k}\right)-h_{1}\left(x^{k-1}\right)\right\|^{q} \\
\leq & \left(\left(1-q v_{1}\right)+\left(C_{q}+q \xi_{1}\right) \delta_{1}^{q}\right)\left\|x^{k}-x^{k-1}\right\|^{q}
\end{aligned}
$$


Substituting (19) in (18), we get

$$
\leq \frac{\left\|x^{k}-x^{k-1}\right\|}{1-\sqrt[q]{\left(1-q v_{1}\right)+\left(C_{q}+q \xi_{1}\right) \delta_{1}^{q}}}\left\|z^{k}-z^{k-1}\right\|
$$

Similarly, we can prove that

$$
\leq \frac{\left\|y^{k}-y^{k-1}\right\|}{1-\sqrt[q]{\left(1-q v_{2}\right)+\left(C_{q}+q \xi_{2}\right) \delta_{2}^{q}}}\left\|t^{k}-t^{k-1}\right\| .
$$

From (16),(17), (20) and (21), it follows that

$$
\begin{aligned}
& \left\|z^{k+1}-z^{k}\right\| \\
\leq & \left(1-\alpha\left(1-\frac{\rho \mu_{1}}{1-\sqrt[q]{\left(1-q v_{1}\right)+\left(C_{q}+q \xi_{1}\right) \delta_{1}^{q}}}\right)\right) \\
& \times\left\|z^{k}-z^{k-1}\right\| \\
& +\frac{\alpha \sqrt[q]{\left(\left(\sigma_{1}^{q}-\rho q \theta_{1}\right)+\left(\rho q \kappa_{1}+C_{q} \rho^{q}\right) \gamma_{1}^{q}\right)}}{1-\sqrt[q]{\left(1-q v_{2}\right)+\left(C_{q}+q \xi_{2}\right) \delta_{2}^{q}}} \\
& \times\left\|t^{k}-t^{k-1}\right\|
\end{aligned}
$$

and

$$
\begin{aligned}
& \left\|t^{k+1}-t^{k}\right\| \\
\leq & \left(1-\alpha\left(1-\frac{\eta \mu_{2}}{1-\sqrt[q]{\left(1-q v_{2}\right)+\left(C_{q}+q \xi_{2}\right) \delta_{2}^{q}}}\right)\right) \\
& \times\left\|t^{k}-t^{k-1}\right\| \\
& +\frac{\alpha \sqrt[q]{\left(\left(\sigma_{2}^{q}-\eta q \theta_{2}\right)+\left(\eta q \kappa_{2}+C_{q} \eta^{q}\right) \gamma_{2}^{q}\right)}}{1-\sqrt[q]{\left(1-q v_{1}\right)+\left(C_{q}+q \xi_{1}\right) \delta_{1}^{q}}} \\
& \times\left\|z^{k}-z^{k-1}\right\|
\end{aligned}
$$

Now, we define $\|\cdot\|_{*}$ on $X \times X$ by $\|(x, y)\|_{*}=\|x\|+\|y\|$, for all $(x, y) \in X \times X$. From (22) and (23), it follows that

$$
\begin{aligned}
& \left\|\left(z^{k+1}, t^{k+1}\right)-\left(z^{k}, t^{k}\right)\right\|_{*} \\
\leq & (1-\alpha)\left\|\left(z^{k}, t^{k}\right)-\left(z^{k-1}, t^{k-1}\right)\right\|_{*} \\
+ & \alpha \Lambda\left\|\left(z^{k}, t^{k}\right)-\left(z^{k-1}, t^{k-1}\right)\right\|_{*}
\end{aligned}
$$

where

$$
\begin{aligned}
& \Lambda=\max \left(\frac{\rho \mu_{1}+\sqrt[q]{\left(\left(\sigma_{2}^{q}-\eta q \theta_{2}\right)+\left(\eta q \kappa_{2}+C_{q} \eta^{q}\right) \gamma_{2}^{q}\right)}}{1-\sqrt[q]{\left(1-q v_{1}\right)+\left(C_{q}+q \xi_{1}\right) \delta_{1}^{q}}},\right. \\
&\left.\frac{\eta \mu_{2}+\sqrt[q]{\left(\left(\sigma_{1}^{q}-\rho q \theta_{1}\right)+\left(\rho q \kappa_{1}+C_{q} \rho^{q}\right) \gamma_{1}^{q}\right)}}{1-\sqrt[q]{\left(1-q v_{2}\right)+\left(C_{q}+q \xi_{2}\right) \delta_{2}^{q}}}\right)
\end{aligned}
$$

In view of the condition (12), we know that $0 \leq \Lambda<1$. Thus it follows from (24) that, for each $k \geq k_{0}$,

$$
\begin{aligned}
& \left\|\left(z^{k+1}, t^{k+1}\right)-\left(z^{k}, t^{k}\right)\right\|_{*} \\
\leq & (1-\alpha(1-\Lambda))\left\|\left(z^{k}, t^{k}\right)-\left(z^{k-1}, t^{k-1}\right)\right\|_{*} \\
\leq & (1-\alpha(1-\Lambda))^{2}\left\|\left(z^{k-1}, t^{k-1}\right)-\left(z^{k-2}, t^{k-2}\right)\right\|_{*} \\
\vdots & \\
\leq & (1-\alpha(1-\Lambda))^{k-k_{0}}\left\|\left(z^{k_{0}+1}, t^{k_{0}+1}\right)-\left(z^{k_{0}}, t^{k_{0}}\right)\right\|_{*} .
\end{aligned}
$$

Hence, for any $m \geq n>k_{0}$, we have

$$
\begin{aligned}
& \left\|\left(z^{m}, t^{m}\right)-\left(z^{n}, t^{n}\right)\right\|_{*} \\
\leq & \sum_{j=n}^{m-1}\left\|\left(z^{j+1}, t^{j+1}\right)-\left(z^{j}, t^{j}\right)\right\|_{*} \\
\leq & \sum_{j=n}^{m-1}(1-\alpha(1-\Lambda))^{j-k_{0}}\left\|\left(z^{k_{0}+1}, t^{k_{0}+1}\right)-\left(z^{k_{0}}, t^{k_{0}}\right)\right\|_{*} .
\end{aligned}
$$

Since $1-\alpha(1-\Lambda)<1$, it follows from (25) that $\left\|\left(z^{m}, t^{m}\right)-\left(z^{n}, t^{n}\right)\right\|_{*}=\left\|z^{m}-z^{n}\right\|+\left\|t^{m}-t^{n}\right\| \rightarrow 0$, as $n \rightarrow \infty$. Hence $\left\{z^{n}\right\},\left\{t^{n}\right\}$ are both Cauchy sequences in $K$ and so there exist $z^{*}, t^{*} \in K$ such that $z^{n} \rightarrow z^{*}$ and $t^{n} \rightarrow t^{*}$ as $n \rightarrow \infty$. From (20) and (21) it follows that the sequences $\left\{x^{n}\right\}$ and $\left\{y^{n}\right\}$ are also both Cauchy Cauchy sequences. Thus there exist $x^{*}, y^{*} \in K$ such that $x^{n} \rightarrow x^{*}$ and $y^{n} \rightarrow y^{*}$ as $n \rightarrow \infty$.

Since the mappings $g_{1}, g_{2}, h_{1}, h_{2}$ and $Q_{K}$ are continuous, it follows from (11) that

$$
h_{1}\left(x^{*}\right)=Q_{K}\left[g_{1}\left(y^{*}\right)-\rho T_{1}\left(y^{*}, x^{*}\right)\right]
$$

and

$$
h_{2}\left(x^{*}\right)=Q_{K}\left[g_{2}\left(x^{*}\right)-\eta T_{2}\left(x^{*}, y^{*}\right)\right] .
$$

Now, Lemma 3.1 guarantees that $\left(x^{*}, y^{*}\right)$ is a solution of the problem (1). This completes the proof.

Theorem 3.2 Let $T_{i}, g_{i}, h_{i}(i=1,2)$ be nonlinear operators, such that for each $\mathrm{i}=1,2$,

(a) $T_{i}$ is $\theta_{i}$-strongly monotone with respect to $g_{i}$ and $\gamma_{i}$-Lipschitz continuous in the first variable, and $\mu_{i}$-Lipschitz continuous in the second variable;

(b) $h_{i}$ is $v_{i}$-strongly monotone and $\delta_{i}$-Lipschitz continuous;

(c) $g_{i}$ is $\sigma_{i}$-Lipschitz continuous.

If there exist constants $\rho, \eta>0$ such that

$$
\left\{\begin{array}{l}
\sqrt[q]{\left(\sigma_{2}^{q}-\eta q \theta_{2}\right)+C_{q} \eta^{q} \gamma_{2}^{q}}<1-k_{1}-\rho \mu_{1}, k_{1}+\rho \mu_{1}<1 \\
\sqrt[q]{\left(\left(\sigma_{1}^{q}-\rho q \theta_{1}\right)+C_{q} \rho^{q} \gamma_{1}^{q}\right)}<1-k_{2}-\eta \mu_{2}, k_{2}+\eta \mu_{2}<1, \\
\text { and } \\
q v_{i}<1+C_{q} \delta_{i}^{q},(i=1,2) \\
\text { where } \\
k_{i}=\sqrt[q]{1-\left(q v_{i}-C_{q} \delta_{i}^{q}\right)},(i=1,2)
\end{array}\right.
$$

then for arbitrarily chosen initial points $x_{0}, y_{0} \in K, x^{k}$ and $y^{k}$ obtained from Algorithm 3.1 converge strongly to $x^{*}$ and $y^{*}$ respectively.

\section{Conclusion}

A new system of extended general variational inequalities in Banach spaces is introduced and studied. It is shown that the new system is equivalent to the system of fixed point problems. This alternative equivalent formulation is used to suggest an iterative method for solving system along with convergence criteria. Some special cases are studied. Results proved in this paper continue for these known and new systems. The implementation and comparison of these methods with other methods is a subject of the future research. 


\section{References}

[1] R. E. Bruck, Nonexpansive projections on subsets of Banach spaces, Pacic J. Math., 47, 341-355 (1973).

[2] S. S. Chang, H. W. Joseph Lee and C. K. Chan, Generalized system for relaxed cocoercive variational inequalities in Hilbert spaces, Appl. Math. Lett., 20, 329-334 (2007).

[3] K. Goebel, S. Reich, Uniform convexity, in: Hyperbolic Geometry, and Nonexpansive Mappings, Marcel Dekker, New York, (1984).

[4] Heng-you Lan, Nonlinear parametric multi-valued variational inclusion systems involving $(A, \eta)$-accretive mappings in Banach spaces, Nonlinear Anal., 69, 17571767 (2008)

[5] Z. Huang and M. A. Noor, An explicit projection method for a system of nonlinear variational inequalities with different $(\gamma, r)$-cocoercive mappings, Appl. Math. Comput., 190, 356-361 (2007).

[6] J. L. Lions and G. Stampacchia, Variational inequalities, Comm. Pure Appl. Math., 20, 493-512 (1967).

[7] A. Moudafi, An algorithmic approach to prox-regular variational inequalities, Appl. Math. Comput., 155, 845-852 (2004).

[8] M. A. Noor, Iterative schemes for nonconvex variational inequalites, J. Optim. Theory Appl., 121, 385-395 (2004).

[9] M. A. Noor, Variational Inequalities and Applications, Lecture Notes, Mathematics Department, COMSATS Institute of information Technology, Islamabad, Pakistan, (2007-2013).

[10] M. A. Noor, K. I. Noor, Projection algorithms for solving system of general variational inequalities, Nonlinear Anal., 70, 2700-2706 (2009).

[11] M. A. Noor, Extended general variational inequalities, Appl. Math. Lett., 22, 182-185 (2009).

[12] D. S. Mitrinovic, J. E. Pecaric, A. M. Fink. Classical and New Inequalities in Analysis. Mathematics and Its Applications (East European Series), 61, (1993)

[13] M. A. Noor, K. I. Noor, Sensitivity analysis of some quasi variational inequalities, J. Adv. Math. Stud., 6, 43-52 (2013).

[14] M. A. Noor, K. I. Noor, A. G. Khan, Some iterative schemes for solving extended general quasi variational inequalities, Appl. Math. Infor. Sci., 7, 917-925 (2013).

[15] L. P. Pang, J. Shen and H. S. Song, A modified predictorcorrector algorithm for solving nonconvex generalized variational inequalities, Comput. Math. Appl., 54, 319-325 (2007).

[16] R. A. Poliquin, R. T. Rockafellar and L. Thibault, Local differentiability of distance functions, Trans. Am. Math. Soc., 352, 5231-5249 (2000).

[17] G. Stampacchia, Formes bilineaires coercitives sur les ensembles convexes, C. R. Acad. Sci. Paris, 258, 4413-4416 (1964) .

[18] R. U. Verma, Projection methods, algorithms, and a new system of nonlinear variational inequalities, Comput. Math. Appl., 41, 1025-1031 (2001).

[19] R. U. Verma, Generalized system for relaxed cocoercive variational inequalities and projection methods, J. Optim. Theory Appl., 121, 203-210 (2004).

[20] R. U. Verma, General convergence analysis for two-step projection methods and applications to variational problems, Appl. Math. Lett., 18, 1286-1292 (2005).
[21] H. K. Xu, Inequalities in Banach spaces with applications, Nonlinear Anal., 16, 1127-1138 (1991).

[22] H. Yang, L. Zhou and Q. Li, A parallel projection method for a system of nonlinear variational inequalities, Appl. Math. Comput., 217, 1971-1975 (2010).

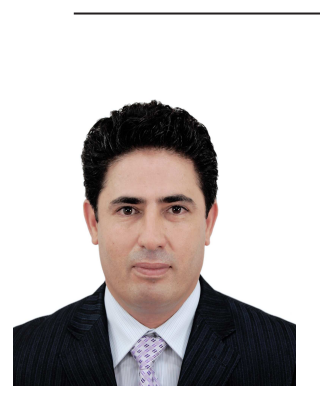

Abdellah Bnouhachem
graduate from Nanjing
university, Nanjing, China.
$\mathrm{He}$ received his Ph.D. degree
in Computational Mathematics
in 2005. He has been honored
by Ministry of Science
and Technology of China
during the launching ceremony
of Partnership Africa and
China in the field of science
and technology on November 24,
2009. He has been awarded SCI Prize, Nanjing University on June 2009. Currently, he is Associate Professor at the University Ibn Zohr, ENSA, Agadir, Morocco.

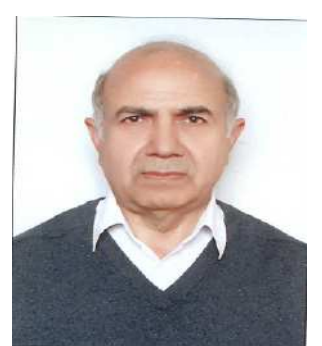

Muhammad

Aslam Noor earned his PhD degree from Brunel University, London, UK (1975) in the field of Applied Mathematics(Numerical Analysis and Optimization). He has vast experience of teaching and research at university levels in various countries including Pakistan, Iran, Canada, Saudi Arabia and UAE. His field of interest and specialization is versatile in nature. It covers many areas of Mathematical and Engineering sciences such as Variational Inequalities, Operations Research and Numerical Analysis. He has been awarded by the President of Pakistan: President's Award for pride of performance on August 14, 2008, in recognition of his contributions in the field of Mathematical Sciences. He was awarded HEC Best Research award in 2009. He is currently member of the Editorial Board of several reputed international journals of Mathematics and Engineering sciences. He has more than 750 research papers to his credit which were published in leading world class journals.

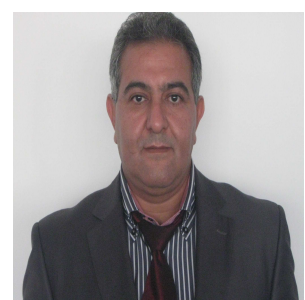




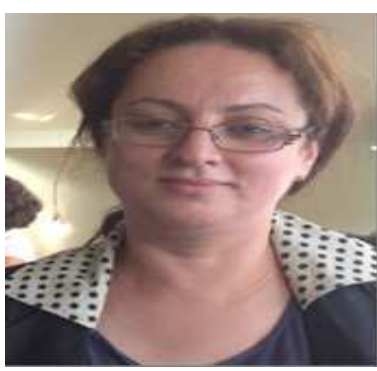

Hafida Benazza graduate from Mohammed V-Agdal University. She obtained her Ph.D. degree in the Automatic and Industrial Informatics (optional: Optimal Control) at Mohammadia School of Engineers in 2004. Currently, she is a Professor at High School of Technology in Sal, Mohammed V-Agdal University, Morocco. 